\title{
O conhecimento sobre Aids de homens idosos e adultos jovens: um estudo sobre a percepção desta doença.
}

\author{
Awareness about Aids among elderly males and young adults: \\ a study of the perception of this disease
}

${ }^{1}$ Departamento de Medicina Social, Universidade Federal de Pernambuco. Av Prof Moraes Rêgo 1235, Cidade Universitária. 50670-420 Recife PE. hugomouramelo@gmail.com

\begin{abstract}
The scope was to compare awareness of elderly males and young adults about aids, taking education into consideration. By means of an epidemiological, descriptive and cross-sectional study, the information of 30 elderly males and 62 young adults about the concept, transmission, prevention, diagnosis and treatment of aids was investigated by structured interviews based on a standardized and validated questionnaire. Frequency distribution, Chi-square test or Fisher's exact test, as well as Student's t test or the MannWhitney test were used, with a significance level of 0.05 for contingency and variance analysis. The Mantel-Haenszel test with a significance level of 0.05 and Odds Ratio with 95\% confidence intervals were used to determine scholarship influence. Individual perception of good health was greater among young adults (61.3\% vs $43.3 \%$ for elderly) and major sexual activity for elderly males was detected ( $80 \%$ vs $62.9 \%$ of young adults). Nevertheless, the elderly males declared adequate knowledge about aids less frequently (26.7\% vs $80.6 \%$ of young adults); prior HIV test (13.3\% vs $24.2 \%$ of young adults) and receiving orientation about aids (36.7\% vs $98.4 \%$ of young adults). Elderly males had insufficient information on HIV/aids, when compared with young adults, highlighting the need to pay closer attention to the elderly population.
\end{abstract}

Key words Aids, Knowledge, Elderly
Resumo O objetivo foi comparar conhecimento de homens idosos ao de adultos jovens sobre Aids, considerando escolaridade. Estudo epidemiológico, descritivo e de corte transversal, em que foram analisadas informações de 30 idosos e 62 adultos jovens sobre conceito, transmissão, prevenção, diagnóstico e tratamento de Aids, investigadas por entrevista estruturada baseada em questionário validado padronizado. Para análise de contingência e de variância, empregaram-se distribuições de frequências e testes de Qui quadrado ou exato de Fisher e teste t de Student ou Mann-Whitney em nível de significância de 0,05. Foram empregados o teste de Mantel-Haenszel, em nível de significância de 0,05, e os Odds Ratio com intervalos de confiança a 95\%, para influência da escolaridade. Constataram-se percepção individual de boa saúde maior em jovens (61,3\% contra 43,3\% dos idosos) e atividade sexual maior em idosos (80\% contra $62,9 \%$ dos jovens). Apesar disso foi menos frequente idosos afirmarem conhecimento satisfatório sobre Aids (26,7\% contra 80,6\% dos jovens); história de teste de HIV (13,3\% contra $24,2 \%$ dos jovens) e ter recebido orientação sobre Aids (36,7\% dos idosos e 98,4\% dos jovens). Os idosos tinham informação insuficiente sobre HIV/ Aids comparados a adultos jovens, reforçando a necessidade de maior atenção à população idosa. Palavras-chave Aids, Conhecimento, Idosos 


\section{Introdução}

No Brasil, no período de 2000 a 2008, a população na faixa de 15 a 64 anos teve um acréscimo de $3,4 \%$, em comparação àquela com 65 anos e mais, cujo aumento correspondeu a $20 \%{ }^{1}$. Nos Estados Unidos da América, no mesmo período, o aumento da população abaixo de 65 anos de idade igualou-se a $8,6 \%$, portanto menor que os $12,8 \%$ verificados entre indivíduos de 65 anos e mais $^{2}$.

O envelhecimento populacional constitui um dos maiores desafios para a saúde pública contemporânea, especialmente em países em desenvolvimento, porque exige que se agregue qualidade aos anos adicionais de vida, de tal forma que as políticas destinadas aos idosos devem levar em conta a manutenção de sua capacidade funcional, a necessidade de autonomia, a elaboração de novos significados para a vida na idade avançada, o incentivando à prevenção, o cuidado e a atenção integral à saúde 3 .

Apesar do estereotipo dominante de "pessoas idosas assexuadas", estudo sobre atividade sexual entre idosos mostra um percentual elevado de sujeitos com mais de 50 anos envolvidos em um ou mais relacionamentos sexuais, já que, desde que não haja a ocorrência de processos patológicos severos, nada impede a continuidade da vida sexual de forma prazerosa ${ }^{4,5}$. Estudo realizado entre 2005 e 2006, envolvendo 3.005 pessoas com idade de 57 a 85 anos, mostrou que $67 \%$ dos homens e $39,5 \%$ das mulheres, na faixa etária de 65 a 74 anos, estavam sexualmente ativos, percentual que se reduziu para $38,5 \%$ dos homens e $16,7 \%$ das mulheres entre 75 e 85 anos de idade ${ }^{6}$.

Apesar do aumento da representatividade dessa faixa etária, só recentemente tem sido dada ênfase às características da epidemia da síndrome da imunodeficiência adquirida (Aids), uma doença sexualmente transmissível, em pessoas com idade mais avançada, pelo aumento na proporção de indivíduos mais velhos infectados com o HIV e o subsequente aumento do número de casos de Aids nesse segmento populacional, além de essa faixa de idade ter no mínimo o sexo desprotegido como um fator de risco para contrair o HIV ${ }^{7}$.

No Brasil, a taxa de incidência da Aids em homens na faixa etária de 50-59 anos passou de 21,5 casos/ 100.000 hab em 2000 , para 27,0 casos/ 100.000 hab, em 2007, enquanto que, na faixa etária de 60 anos e mais, aumentou de 6,8 casos/ 100.000 hab, em 2000, para 9,3 casos/100.000 hab, em $2007^{8}$. Tal contexto pode revelar a forma excludente como vem sendo abordada a sexualidade de pessoas maiores de 60 anos de idade, já que só a partir de 2008 tem sido alvo de Programa Nacional de Educação e Prevenção da Aids ${ }^{8}$.

Muito embora uma pequena parcela dos idosos tenha discutido com seus médicos sobre o risco de infecção pelo HIV ou outras DST ${ }^{9}$, essas estatísticas suscitam questionamentos. A relutância dos médicos em discutir com o idoso sobre saúde sexual ou o tempo limitado para a interação médico-paciente durante as consultas ${ }^{10}$ podem dificultar uma abordagem mais profícua? Os idosos realmente diferenciam-se dos jovens quanto ao comportamento sexual no que se refere à prevenção de HIV/Aids? O fator idade e os valores socioculturais/educacionais podem dificultar a adoção de comportamentos preventivos? O aumento da incidência de infecção por HIV/Aids em idosos tem base nas informações que eles detêm sobre a doença?

Poucos estudos investigam o nível de conhecimento de pessoas idosas em relação à Aids e os fatores que influenciam o provável desconhecimento deste segmento populacional sobre a doença. Mesmo em amostras robustas, nas quais existem idosos de ambos os sexos, não são investigados os níveis de conhecimento do segmento mais envelhecido a respeito da transmissão e do tratamento da Aids ${ }^{11}$.

Partindo da comprovação de que a escolaridade é considerada um importante determinante da situação de saúde dos indivíduos, tendo sido evidenciado, por Fonseca et al. ${ }^{12}$, que, no Brasil, a epidemia de Aids se iniciou nos estratos sociais de maior escolaridade, o objetivo deste artigo foi comparar a percepção de homens idosos à de adultos jovens sobre Aids, considerando a escolaridade.

\section{Métodos}

Realizou-se estudo epidemiológico, descritivo, de corte transversal, entre agosto de 2006 e julho de 2007 , envolvendo dois grupos. O primeiro foi formado por todos os participantes do sexo masculino dos cinco grupos de convivência vinculados à Federação das Associações de Idosos de Pernambuco, num total de 30 indivíduos, com idade de 60 anos ou mais, caracterizados como idosos de acordo com a Lei n ${ }^{\circ}$. 8842/94, que dispõe sobre a Política Nacional do Idoso ${ }^{13}$.

Para determinar o grupo de comparação, admitiu-se um segundo, constituído por 62 estu- 
dantes do sexo masculino, que correspondiam ao total do corpo discente dos cursos pré-vestibulares ("Vestibular Cidadão" e "Portal"), oferecidos por estudantes de Direito e Medicina da Universidade Federal de Pernambuco. Este grupo se caracterizava por estar na faixa etária de 18 a 29 anos e apresentar escolaridade mais homogênea em virtude de ter concluído o $2^{\circ}$ grau e estar sendo submetido a reforço de conhecimentos.

Para coleta dos dados, empregou-se entrevista estruturada, com base em questionário, contendo variáveis sociodemográficas de descrição amostral, incluindo idade, escolaridade, situação conjugal, percepção individual de saúde, nível de satisfação com a vida e com a vida sexual, enquanto que as variáveis relativas ao nível de conhecimento sobre Aids foram baseadas no protocolo do Projeto Epidoso (Epidemiologia do Envelhecimento) - Estudo Longitudinal com uma população de idosos residentes no município de São Paulo, validado para a população idosa brasileira por Vieira et al. ${ }^{14}$ e no questionário proposto por Batista ${ }^{15}$, e incluíram: conceito da enfermidade (conceito e agente etiológico), transmissão (uso de drogas injetáveis, transfusão sanguínea e opção sexual), prevenção (meios adotados e orientações recebidas) e diagnóstico/tratamento (ser submetido à pesquisa de HIV, existência de tratamento e possibilidade de cura).

As respostas subjetivas foram consideradas satisfatórias quando incluíram: no conceito de Aids, doença que afeta a imunidade ou doença sexualmente transmissível; na transmissão, transmissibilidade por sexo, transfusão ou reutilização/compartilhamento de seringas; e, na prevenção, a referência ao uso do preservativo.

Os dados foram organizados e analisados por meio do programa Statistical Package for Social Sciences, versão 13.0. Empregaram-se distribuições de frequências absolutas e relativas, e os testes de Qui quadrado ou exato de Fisher, t de Student ou U de Mann-Whitney, todos em nível de significância de 0,05. Admitindo que o nível de escolaridade poderia interferir na percepção da AIDS, considerou-se o subgrupo de idosos e jovens adultos com escolaridade a partir de ensino médio completo, porque este foi o menor nível entre jovens adultos. Foi empregado o teste de Mantel-Haenszel em nível de significância de 0,05 e os Odds Ratio (OR) com intervalos de confiança a 95\% (IC95\%).

O projeto de pesquisa foi aprovado pelo Comitê de Ética do Centro de Ciências da Saúde da Universidade Federal de Pernambuco e os integrantes das duas populações foram convidados a participar da pesquisa, após lhes terem sido explicados os objetivos.

\section{Resultados}

Dentre os idosos que responderam às informações sócio-demográficas, houve predomínio na faixa etária de 60 a 69 anos (50\%), morando com companheira $(57,1 \%)$ e com escolaridade fundamental ou de nível médio incompleto $(46,4 \%)$ (Tabela 1). No grupo de adultos jovens, predominou a faixa etária de 18 a 21 anos (54,8\%), solteiros e sem companheira fixa $(90,2 \%)$, com escolaridade de nível médio (100\%) (Tabela 1).

Nas variáveis relativas à saúde geral e hábitos sexuais, constatou-se que $80 \%$ dos idosos perceberam sua saúde como ótima ou boa, percentual menor que $98,4 \%$ dos adultos jovens com essa percepção. Entre os idosos, foi mais frequente considerar a saúde como má ou péssima. Essas diferenças foram significantes $(\mathrm{p}=0,004)$ (Tabela 2).

Os dois grupos apresentaram características diferentes, embora não significantes. Idosos mais frequentemente consideraram médio seu nível de satisfação com a vida $(55,2 \%)$ enquanto que para adultos jovens essa satisfação teve nível alto $(58,1 \%)$. Na faixa etária de 15 a 20 anos, idosos e adultos jovens mais frequentemente tiveram a coitarca, porém foi nos extremos de idade que os dois grupos diferiram: idosos declararam coitarca antes dos 15 anos de idade e adultos jovens após os 20 anos. Para os indivíduos sexualmente ativos, o tipo de parceiro sexual se associou significantemente ao grupo, sendo fixo(a) entre os idosos $(87,5 \%)$ e eventual, conhecido(a) ou desconhecido(a) entre os adultos jovens $(\mathrm{p}<0,001)$ (Tabela 2).

Em relação ao conhecimento sobre Aids (Tabela 3), constatou-se que o conceito de Aids manteve associação significante com o grupo, porque as respostas insatisfatórias para o conceito da enfermidade $(p<0,001)$ e do agente etiológico $(\mathrm{p}=0,038)$ predominaram entre os idosos, quando comparados aos adultos jovens. Analogamente, o conhecimento sobre a transmissão da Aids associou-se ao grupo, porque os idosos mais frequentemente omitiram a informação ou forneceram respostas insatisfatórias quanto à forma de contágio, quando comparados aos adultos jovens $(\mathrm{p}=0,026)$ (Tabela 3$)$.

Houve associação significante do conhecimento de prevenção de Aids entre os grupos. O conhecimento de uso correto e a prática do uso de condom foram características dos adultos jo- 
Tabela 1. Distribuição de variáveis sociodemográficas de idosos e adultos jovens - Recife, Agosto de 2006 Julho de 2007

\begin{tabular}{|c|c|c|c|c|}
\hline \multirow{3}{*}{ Variáveis sociodemográficas } & \multicolumn{4}{|c|}{ Grupos de estudo } \\
\hline & \multicolumn{2}{|c|}{ Idosos $(n=30)$} & \multicolumn{2}{|c|}{ Adultos jovens $(n=62)$} \\
\hline & $\mathbf{n}$ & $\%$ & $\mathbf{n}$ & $\%$ \\
\hline \multicolumn{5}{|l|}{ Idade $(\operatorname{anos})^{1}$} \\
\hline $18-21$ & - & - & 34 & 54,8 \\
\hline $22-25$ & - & - & 18 & 29,0 \\
\hline $26-29$ & - & - & 10 & 16,2 \\
\hline $60-69$ & 14 & 50,0 & - & - \\
\hline $70-89$ & 12 & 42,9 & - & - \\
\hline$\geq 90$ & 2 & 7,1 & - & - \\
\hline \multicolumn{5}{|l|}{ Situação conjugal ${ }^{2}$} \\
\hline Solteiro ou sem companheira & 12 & 42,9 & 55 & 90,2 \\
\hline Com companheira & 17 & 57,1 & 6 & 9,8 \\
\hline \multicolumn{5}{|l|}{ Escolaridade $^{1}$} \\
\hline Iletrado ou fundamental incompleto & 6 & 21,4 & - & - \\
\hline Fundamental ou médio incompleto & 13 & 46,4 & - & - \\
\hline Médio ou superior incompleto & 7 & 25,0 & 62 & 100,0 \\
\hline Superior completo & 2 & 7,2 & - & - \\
\hline
\end{tabular}

${ }^{1}$ - Dois idosos (6,7\%) omitiram a informação relativa a idade e escolaridade. ${ }^{2}$ - Um idoso (3,3\%) e 1 (1,6\%) adulto jovem omitiu a situação conjugal

vens, porque os idosos declararam não saber usálo $(65,5 \%)(\mathrm{p}<0,001)$ e não ter essa prática como hábito $(70 \%)(\mathrm{p}<0,001) ; 53,3 \%$ dos idosos forneceram respostas insatisfatórias quanto às condutas preventivas, contra $11,3 \%$ dos adultos jovens $(\mathrm{p}<0,001)$ (Tabela 3$)$.

Mais frequentemente os jovens julgaram ter adotado condutas de risco para contrair Aids $(22 \%)$, quando comparados aos idosos $(3,3 \%)$, diferença que foi significante $(\mathrm{p}=0,030)$.

A importância do diagnóstico de Aids, assim como o conhecimento sobre tratamento foram características que se associaram significantemente ao grupo. Os adultos jovens, comparados aos idosos, mais frequentemente julgaram importante se submeter ao teste $(\mathrm{p}<0,001)$ e reconheceram a existência de tratamento para Aids $(\mathrm{p}<0,001)$.

Questionados os dois grupos quanto à possibilidade de uma pessoa com aparência saudável poder ser portadora de Aids, identificou-se que mais frequentemente os idosos discordaram $(36,7 \%)$, contrapondo-se aos adultos jovens que admitiram essa possibilidade $(98,4 \%)$, diferença que foi significante $(\mathrm{p}<0,001)$.

Para avaliar a influência da escolaridade sobre a percepção da saúde geral e dos hábitos sexuais, foram comparados idosos e adultos jo- vens com mesmo nível de escolaridade (igual ou maior que $2^{\circ}$ grau completo), visto não ter havido adultos jovens com escolaridade menor. Foi possível identificar que os idosos perceberam o estado de saúde e o nível de satisfação com a vida diferente do que o fizeram os adultos jovens, e os Odds Ratio foram significantes (Tabela 4). A comparação dos dados das Tabelas 2 e 4 permitiu constatar a existência de influência da escolaridade no nível de satisfação com a vida e na escolha do tipo de parceiro(a) sexual, porque houve mudança na significância das diferenças. A percepção de saúde geral independeu da escolaridade.

Identificou-se que os adultos jovens pensavam diferente dos idosos, com mesmo nível de escolaridade, com relação ao conhecimento de Aids e de condutas preventivas, orientação sobre prevenção de Aids e importância do teste de HIV, com Odds Ratio significantes. Comparando os resultados das Tabelas 3 e 5, constatou-se que o conhecimento de Aids e de condutas preventivas, orientação sobre a prevenção de Aids, ter se submetido à pesquisa de HIV, importância do teste de HIV e possibilidade de cura independeram da escolaridade, visto ter havido concordância dos resultados. No entanto conhecimento do agente etiológico da Aids, transmissibilidade da doença, conhecimento e referência do uso do condom, ado- 
Tabela 2. Distribuição de variáveis relativas à saúde geral e hábitos sexuais de idosos e adultos jovens Recife, Agosto de 2006 - Julho de 2007

\begin{tabular}{|c|c|c|c|c|c|}
\hline \multirow{3}{*}{$\begin{array}{c}\text { Variáveis relativas à saúde geral } \\
\text { e hábitos sexuais }\end{array}$} & \multicolumn{4}{|c|}{ Grupos de estudo } & \multirow{3}{*}{ Valor de $\mathrm{p}$} \\
\hline & \multicolumn{2}{|c|}{ Idosos $(n=30)$} & \multicolumn{2}{|c|}{ Adultos jovens $(n=62)$} & \\
\hline & $\mathbf{n}$ & $\%$ & n & $\%$ & \\
\hline \multicolumn{6}{|l|}{ Percepção de saúde } \\
\hline Ótima & 11 & 36,7 & 23 & 37,1 & $\mathrm{p}=0,004$ \\
\hline Boa & 13 & 43,3 & 38 & 61,3 & \\
\hline Má & 4 & 13,3 & 1 & 1,6 & \\
\hline Péssima & 2 & 6,7 & - & - & \\
\hline Nível de satisfação com a vida ${ }^{1}$ & & & & & $\mathrm{p}=0,073$ \\
\hline Alto & 11 & 37,9 & 36 & 58,1 & \\
\hline Médio & 16 & 55,2 & 24 & 38,7 & \\
\hline Baixo & 2 & 6,9 & 2 & 3,2 & \\
\hline Opção sexual ${ }^{2}$ & & & & & $\mathrm{p}=0,226$ \\
\hline Heterossexual & 25 & 92,6 & 59 & 93,3 & \\
\hline Homossexual & 1 & 3,7 & 1 & 6,7 & \\
\hline Bissexual & 1 & 3,7 & - & - & \\
\hline Idade à coitarca $(\text { anos })^{3}$ & & & & & $\mathrm{p}=0,136$ \\
\hline$<15$ & 8 & 26,7 & 7 & 12,5 & \\
\hline $15-20$ & 20 & 66,7 & 39 & 69,6 & \\
\hline$>20$ & 2 & 6,7 & 10 & 17,9 & \\
\hline Atividade sexual & & & & & $\mathrm{p}=0,098$ \\
\hline Ativo & 24 & 80,0 & 39 & 62,9 & \\
\hline Inativo & 6 & 20,0 & 23 & 37,1 & \\
\hline Tipo de parceiro sexual ${ }^{4}$ & & & & & $\mathrm{p}<0,001$ \\
\hline Companheiro(a) fixo(a) & 21 & 87,5 & 10 & 26,3 & \\
\hline Parceiro(a) eventual conhecido(a) & 2 & 8,3 & 23 & 60,5 & \\
\hline Parceiro(a) eventual desconhecido(a) & 1 & 4,2 & 5 & 13,2 & \\
\hline
\end{tabular}

ção de condutas de risco, existência de tratamento para Aids e possibilidade de um indivíduo com aparência saudável ter Aids dependeram da escolaridade, porque ao homogeneizar os grupos, houve mudança na significância das diferenças.

\section{Discussão}

Observou-se menor conhecimento dos idosos sobre Aids, quando comparados aos jovens, perpassando o conceito da enfermidade, transmissibilidade, condutas preventivas, diagnóstico e tratamento, pois ofereceram maior número de respostas insatisfatórias. Em Henderson et al. ${ }^{16} \mathrm{e}$ Zablotsky e Kennedy ${ }^{17}$, o conhecimento sobre Aids mostrou-se associado de forma inversa ao aumento da idade, sendo menor entre os idosos, quando comparados aos indivíduos mais jovens, assim como no presente estudo.
Knodel e Zimmer ${ }^{18}$, analisando o conhecimento de idosos sobre Aids no Camboja, julgaram como ponto mais surpreendente da pesquisa a similaridade do menor conhecimento de Aids entre idosos, quando comparados aos jovens, em países economicamente e educacionalmente tão díspares quanto a Tailândia e os Estados Unidos. Zornitta ${ }^{19}$ pareceu fornecer a melhor explicação para esse fato ao afirmar que historicamente a sexualidade dos idosos tem sido negada, ela é anulada na sua dimensão, na sua subjetividade, ao longo de construções de estereótipos negativos.

A vulnerabilidade dos idosos às DST e Aids é atribuída, no estudo de Figueiredo e Provinciali ${ }^{20}$, ao desconhecimento da sexualidade dos idosos por parte da sociedade, em geral, e dos profissionais de saúde, em especial. Na sociedade ocidental, a sexualidade dos idosos é dominada pelo pensamento estereotipado, pela ignorância 
Tabela 3. Distribuição das variáveis relativas ao conhecimento sobre Aids entre idosos e adultos jovens Recife, Agosto de 2006 - Julho de 2007

\begin{tabular}{|c|c|c|c|c|c|}
\hline \multirow{3}{*}{$\begin{array}{l}\text { Variáveis relativas } \\
\text { ao conhecimento sobre Aids }\end{array}$} & \multicolumn{4}{|c|}{ Grupos de estudo } & \multirow{3}{*}{ Valor de $p$} \\
\hline & \multicolumn{2}{|c|}{$\operatorname{Idosos}(n=30)$} & \multicolumn{2}{|c|}{ Adultos jovens $(n=62)$} & \\
\hline & $\mathbf{n}$ & $\%$ & $\mathbf{n}$ & $\%$ & \\
\hline \multicolumn{6}{|l|}{ Conceito de Aids } \\
\hline Conceito da enfermidade & & & & & $\mathrm{p}<0,001$ \\
\hline Satisfatório & 8 & 26,7 & 50 & 80,6 & \\
\hline Insatisfatório ou incorreto & 22 & 73,3 & 12 & 19,4 & \\
\hline Agente etiológico & & & & & $\mathrm{p}=0,038$ \\
\hline HIV & 12 & 40,0 & 39 & 62,9 & \\
\hline Resposta insatisfatória & 18 & 60,0 & 23 & 27,1 & \\
\hline Transmissão & & & & & $p=0,026$ \\
\hline \multicolumn{6}{|l|}{ Transmissibilidade da doença } \\
\hline Resposta satisfatória & 21 & 70,0 & 55 & 88,7 & \\
\hline Resposta insatisfatória & 9 & 30,0 & 7 & 11,3 & \\
\hline Prevenção & & & & & $\mathrm{p}<\mathbf{0 , 0 0 1}$ \\
\hline \multicolumn{6}{|l|}{ Conhecimento sobre o uso do condom } \\
\hline Sabe usar & 10 & 34,5 & 60 & 96,8 & \\
\hline Não sabe usar & 19 & 65,5 & 2 & 3,2 & \\
\hline Referência ao uso de condom ${ }^{1}$ & & & & & $\mathrm{p}<0,001$ \\
\hline Sim & 9 & 30,0 & 47 & 87,0 & \\
\hline Não & 21 & 70,0 & 8 & 23,0 & \\
\hline Conhecimento de condutas preventivas ${ }^{2}$ & & & & & $\mathrm{p}<0,001$ \\
\hline Resposta satisfatória & 14 & 46,7 & 55 & 88,7 & \\
\hline Resposta insatisfatória & 16 & 53,3 & 5 & 11,3 & \\
\hline Orientação sobre prevenção de Aids & & & & & $\mathrm{p}<0,001$ \\
\hline Recebeu & 11 & 36,7 & 61 & 98,4 & \\
\hline Não recebeu & 19 & 63,3 & 1 & 1,6 & \\
\hline Adoção de condutas de risco para Aids $^{2}$ & & & & & $\mathrm{p}=\mathbf{0 , 0 3 0}$ \\
\hline Sim & 1 & 3,3 & 13 & 22,0 & \\
\hline Não & 29 & 96,7 & 47 & 78,0 & \\
\hline \multicolumn{6}{|l|}{ Diagnóstico e Tratamento } \\
\hline Foi submetido à pesquisa de HIV & & & & & $\mathrm{p}=0,228$ \\
\hline Sim & 4 & 13,3 & 15 & 24,2 & \\
\hline Não & 26 & 86,7 & 47 & 75,8 & \\
\hline Importância do teste & & & & & $\mathrm{p}<0,001$ \\
\hline Tem & 11 & 36,7 & 59 & 95,2 & \\
\hline Não tem & 19 & 63,3 & 3 & 4,8 & \\
\hline Tratamento para Aids & & & & & $\mathrm{p}<0,001$ \\
\hline Existe & 9 & 31,0 & 48 & 77,4 & \\
\hline Não existe & 21 & 69,0 & 14 & 22,6 & \\
\hline Possibilidade de cura da Aids & & & & & $\mathrm{p}=0,535$ \\
\hline $\operatorname{Sim}$ & 3 & 10,0 & 10 & 16,1 & \\
\hline Não & 27 & 90,0 & 52 & 83,9 & \\
\hline
\end{tabular}

${ }^{1}$ - Sete adultos jovens $(11,3 \%)$ omitiram a informação relativa ao uso de condom. ${ }^{2}$ - Dois adultos jovens $(3,2 \%)$ omitiram as informações sobre conhecimento de condutas preventivas e adoção de condutas de risco para Aids

e pelo preconceito, o que pode influenciar negativamente também o processo de anamnese nas consultas $^{7,21}$. Dentre as concepções errôneas estão admitir que as pessoas idosas não estão interessadas em sexo; se acaso estiverem interessadas, ninguém está interessado nelas; fazem sexo num contexto de relacionamento heterossexual e monogâmico e não são usuárias de droga ${ }^{20}$.

No entanto, na presente pesquisa, $80 \%$ dos idosos se declararam sexualmente ativos e com uma percepção de saúde variando entre boa e ótima, mas apenas 57,1\% tinham companheira. 
Tabela 4. Distribuição das variáveis relativas à saúde geral e hábitos sexuais entre idosos e adultos jovens com escolaridade mínima de $2^{\circ}$ grau completo - Recife, Agosto de 2006 - Julho de 2007

\begin{tabular}{|c|c|c|c|c|c|c|}
\hline \multirow{3}{*}{$\begin{array}{l}\text { Variáveis relativas à saúde } \\
\text { geral e hábitos sexuais }\end{array}$} & \multicolumn{4}{|c|}{ Grupos de estudo } & \multirow{3}{*}{$\begin{array}{l}\text { OR } \\
\text { IC95\% }\end{array}$} & \multirow{3}{*}{$\mathbf{p}^{*}$} \\
\hline & \multicolumn{2}{|c|}{ Idosos $(n=9)$} & \multicolumn{2}{|c|}{ Adultos jovens $(n=62)$} & & \\
\hline & $\mathbf{n}$ & $\%$ & $\mathrm{n}$ & $\%$ & & \\
\hline Percepção de saúde & & & & & $48,8(3,8-1419,5)$ & $<0,001$ \\
\hline Ótima ou boa & 5 & 55,6 & 61 & 98,4 & & \\
\hline Má ou péssima & 4 & 44,4 & 1 & 1,6 & & \\
\hline Nível de satisfação com a vida & & & & & $1,3(1,3-250,8)$ & 0,008 \\
\hline Alto & 1 & 11,1 & 36 & 58,1 & & \\
\hline Médio ou baixo & 8 & 88,9 & 26 & 41,9 & & \\
\hline Opção sexual & & & & & $5,6(0,5-54,7)$ & 0,058 \\
\hline Heterossexual & 7 & 77,8 & 59 & 95,2 & & \\
\hline Homossexual ou bissexual & 2 & 22,2 & 3 & 4,8 & & \\
\hline Idade à coitarca (anos) & & & & & $0,0(00-1,9)$ & 0,086 \\
\hline $15-20$ & 9 & 100,0 & 46 & 74,2 & & \\
\hline$\geq 20$ & - & - & 16 & 25,8 & & \\
\hline Atividade sexual & & & & & $0,8(0,1-4,4)$ & 0,570 \\
\hline Ativo & 6 & 66,7 & 39 & 62,9 & & \\
\hline Inativo & 3 & 33,3 & 23 & 37,1 & & \\
\hline Tipo de parceiro(a) sexual & & & & & - & 0,003 \\
\hline fixo(a) & 5 & 83,3 & 10 & 26,3 & - & \\
\hline $\begin{array}{l}\text { eventual conhecido(a) ou } \\
\text { desconhecido(a) }\end{array}$ & 0 & 0 & 28 & 73,7 & - & \\
\hline
\end{tabular}

- Teste de Mantel-Haenszel

Segundo Lisboa ${ }^{22}$, o aumento do número de idosos contaminados pelas DST e Aids é devido à capa de invisibilidade da população no que concerne a enxergá-los como sujeitos desejantes e sexualmente ativos. No entanto, com o envelhecimento da população, no momento de aumento da expectativa de vida e de novas tecnologias como as que prolongam a vida sexual, esta sexualidade até então ignorada, emerge como um problema de saúde pública, ou seja, a sexualidade dos idosos vem à tona, não pela sua negligência ou anulação, mas pela doença que é o HIV/Aids ${ }^{19}$.

Ao serem questionados sobre o conhecimento em relação ao uso de preservativos, a maioria dos idosos declarou não saber usar assim como o não uso do condom, no que diferiram significantemente dos jovens.

Zawacki et al..$^{23}$, dentre outros fatores, atribuíram o desconhecimento à familiaridade com o parceiro sexual. Para os idosos, a convivência de longos anos traz consigo a familiaridade, a qual pode influenciar as percepções de risco de contaminação pelo HIV pelo viés de projeção, que consiste na tendência de admitir similaridade entre os familiares. Estar próximo pode atuar como um pressuposto de que o parceiro sexual tem baixo risco de estar contaminado pelo HIV e, desse modo, o uso do condom não se faz necessário.

$\mathrm{Na}$ presente pesquisa, constatou-se o perigo dessas pré-concepções dos idosos, já que 3,7\% deles declararam ser homossexual ou bissexual e $12,5 \%$ tinham parceiro(a) eventual conhecido(a) ou desconhecido(a).

A população que vivencia a senectude nos dias atuais não viveu o apelo ao uso do preservativo, como evidenciado atualmente. Os trabalhos educativos, em sua maioria, continuam sendo direcionados ao público jovem, à gestante, ao usuário de droga, aos homossexuais e às profissionais do sexo, o que concorre para que os idosos não se percebam vulneráveis e não incorporem a necessidade de usar o condom ${ }^{24}$.

A decisão pelo uso ou não do preservativo nas relações homem-mulher é, muitas vezes, ainda dependente da anuência do homem e, segundo Geluda et al. ${ }^{25}$, nem sempre pode ser discutida e negociada antes da relação sexual, o que coloca a mulher, que precisa negociar seu uso, numa 
Tabela 5. Distribuição das variáveis relativas ao conhecimento sobre Aids entre idosos e adultos jovens com escolaridade mínima de $2^{\circ}$ grau completo - Recife, Agosto de 2006 - Julho de 2007

\begin{tabular}{|c|c|c|c|c|}
\hline \multirow{3}{*}{$\begin{array}{l}\text { Variáveis relativas ao } \\
\text { conhecimento sobre Aids }\end{array}$} & \multicolumn{2}{|c|}{ Grupos de estudo } & \multirow{3}{*}{$\begin{array}{c}\text { OR } \\
\text { IC95\% }\end{array}$} & \multirow{3}{*}{$\mathbf{p}^{*}$} \\
\hline & $\operatorname{Idosos}(n=9)$ & Adultos jovens $(n=62)$ & & \\
\hline & n (\%) & n (\%) & & \\
\hline Conceito de Aids & & & & $<0,001$ \\
\hline Satisfatório & $5(55,6)$ & $60(96,8)$ & $24,0(2,7-265,6)$ & \\
\hline Insatisfatório ou incorreto & $4(44,4)$ & $2(3,2)$ & & \\
\hline Agente etiológico da Aids & & & & 0,674 \\
\hline HIV & $5(55,6)$ & $39(62,9)$ & $1,4(0,3-6,7)$ & \\
\hline Resposta insatisfatória & $4(44,4)$ & $23(37,1)$ & & \\
\hline Transmissibilidade da doença & & & & 0,275 \\
\hline Resposta satisfatória & $8(88,9)$ & $60(96,8)$ & $3,5(0,0-64,5)$ & \\
\hline Resposta insatisfatória & $1(11,1)$ & $2(3,2)$ & & \\
\hline $\begin{array}{l}\text { Conhecimento sobre o uso do } \\
\text { condom }\end{array}$ & & & $0,21(0,04-1,35)$ & 0,055 \\
\hline Sabe usar & $5(55,6)$ & $47(85,4)$ & & \\
\hline Não sabe usar & $4(44,4)$ & $8(14,6)$ & & \\
\hline Referência ao uso de condom & & & $0,4(0,1-2,33)$ & 0,186 \\
\hline $\operatorname{Sim}$ & $5(55,6)$ & $47(75,8)$ & & \\
\hline Não & $4(44,4)$ & $15(24,2)$ & & \\
\hline $\begin{array}{l}\text { Conhecimento de condutas } \\
\text { preventivas }\end{array}$ & & & $7,4(1,4-42,4)$ & 0,004 \\
\hline Resposta satisfatória & $4(44,4)$ & $53(85,5)$ & & \\
\hline Resposta insatisfatória & $5(55,6)$ & $9(14,5)$ & & \\
\hline $\begin{array}{l}\text { Orientação sobre prevenção de } \\
\text { Aids }\end{array}$ & & & $48,8(3,8-1419,5)$ & $<0,001$ \\
\hline Recebeu & $5(55,6)$ & $61(98,4)$ & & \\
\hline Não recebeu & $4(44,4)$ & $1(1,6)$ & & \\
\hline Adoção de condutas de risco & & & $2,1(0,2-49,3)$ & 0,490 \\
\hline Sim & $1(11,1)$ & $13(21,0)$ & & \\
\hline Não & $8(88,9)$ & $49(79,0)$ & & \\
\hline Foi submetido ao teste de HIV & & & $1,1(0,2-8,8)$ & 0,898 \\
\hline Sim & $2(22,2)$ & $15(24,2)$ & & \\
\hline Não & $7(77,8)$ & $47(75,8)$ & & \\
\hline Importância do teste & & & $24,6(3,3-218,4)$ & $<0,001$ \\
\hline Tem & $4(44,4)$ & $59(95,2)$ & & \\
\hline Não tem & $5(55,6)$ & $3(4,8)$ & & \\
\hline Tratamento para Aids ${ }^{\ddagger}$ & & & $3,4(0,6-19,4)$ & 0,097 \\
\hline Existe & $4(50,0)$ & $48(77,4)$ & & \\
\hline Não existe & $4(50,0)$ & $14(22,6)$ & & \\
\hline Possibilidade de cura da Aids & & & $0,7(0,1-5,5)$ & 0,651 \\
\hline Sim & $2(22,2)$ & $10(16,1)$ & & \\
\hline Não & $7(77,8)$ & $52(83,9)$ & & \\
\hline Aparência saudável e Aids & & & $7,6(0,0-318,1)$ & 0,110 \\
\hline Podem coexistir & $8(88,9)$ & $61(98,4)$ & & \\
\hline Não podem coexistir & $1(11,1)$ & $1(1,6)$ & & \\
\hline
\end{tabular}

* Teste de Mantel-Haenszel. ${ }^{\dagger}-$ Sete adultos jovens omitiram a resposta quanto ao conhecimento do uso do condom. ${ }^{\ddagger}$ - Um idoso omitiu a informação sobre tratamento de Aids

situação desprivilegiada em que fica evidente a importância do trabalho educativo com essa população para o controle da Aids.
Em Guerriero et al. ${ }^{26}$, o uso do preservativo entre os homens entrevistados não era frequente, pois eles acreditavam diminuir o prazer e pre- 
judicar a ereção. Foi ainda referido que os homens não consideravam legítimo que as esposas solicitassem o uso da camisinha para prevenção da Aids, pois deveriam confiar nos maridos. Além disso, foi observado que muitos não sabiam colocar corretamente o preservativo nem tinham conhecimento de que a camisinha tem prazo de validade e de que deve ser protegida do calor, ratificando a deficiência de informações acerca dos meios de prevenção da Aids.

Diferente da opinião de Guerriero et al. ${ }^{26}$, outros autores admitem que a negociação para uso dos preservativos é difícil para ambos os sexos e em todas as faixas etárias. Silva et al. ${ }^{24}$ comentam que as escolhas dependem de um conjunto de fatores correlatos e não somente do risco aparente. O problema vai além de usar ou não o condom e passa por questões maiores ligadas à sexualidade, construção social, mudança de hábitos e promoção da saúde. Segundo Berquó et al. ${ }^{27}$, a compreensão das diferenças quanto ao uso de preservativos requer análises específicas para cada um dos sexos, pois as escolhas estão intimamente conectadas à existência de diferenças de sexo no que se refere às percepções de vínculo afetivo-sexual e à necessidade de proteção.

Silva e Sá ${ }^{11}$ e Inelmen et al. ${ }^{7}$ corroboram Guerriero et al. ${ }^{26}$ ao relatarem os vários obstáculos ao uso da camisinha pelo homem, que muitas vezes julga ser o cuidado necessário apenas em relações extraconjugais ou com profissionais do sexo e para uso exclusivo como método contraceptivo (desconsiderando, neste caso, o potencial de proteção contra DST).

Para conferir maior fidedignidade aos resultados desta pesquisa, buscou-se obedecer à distribuição etária populacional brasileira, pois os dados do IBGE confirmam uma maior proporção de idosos entre 60 e 69 anos e de estudantes, com ensino médio completo, na faixa etária dos 18 aos 21 anos $^{2}$.

No entanto, é preciso ressaltar que não apenas aos idosos são necessárias mais informações sobre Aids, posto que $19,4 \%$ dos adultos jovens não sabiam o que é; $27,1 \%$ desconheciam seu agente etiológico, o que parece reforçar a necessidade de detalhamento de questionários para investigação do conhecimento sobre Aids, para que essas dúvidas possam ser empregadas nas campanhas de educação para a saúde, direcionando as informações de forma mais adequada.

Os dados da presente pesquisa apontaram que os idosos têm informações insuficientes sobre a transmissão sexual do HIV, tal como referido por Henderson et al. ${ }^{16}$, que alertaram para a necessidade de não perder de vista essa parcela da população quando são discutidas políticas de prevenção às DST.

Além das argumentações empregadas nesta discussão, a escolaridade é outro fator que deve ser valorizado. O nível educacional é reconhecido, não apenas como uma expressão das diferenças de acesso à informação e das perspectivas e possibilidades de se beneficiar com novos conhecimentos, mas tem grande importância como determinante de saúde. É estável ao longo dos anos, diferente de outras variáveis socioeconômicas $^{1}$. Esta importância da escolaridade pode explicar as diferenças verificadas no presente estudo entre a análise dos resultados gerais e aquela realizada ao comparar o grupo de idosos ao de adultos jovens, todos, no mínimo, com segundo grau completo, mas essa constatação deve ser interpretada com cautela.

Fonseca et al. ${ }^{12}$ afirmaram que a escolaridade do idoso, expressa em anos de estudo, mostrou-se como variável mais relevante após a idade, na percepção de saúde, o que não se verificou neste estudo, porque adultos jovens e idosos perceberam seu estado de saúde independente da escolaridade.

No entanto a identificação de que as variáveis relacionadas ao conhecimento da Aids e de sua prevenção sofreram influência da escolaridade pareceu confirmar o alerta de Fonseca et al. ${ }^{12}$ quanto à importância desta variável. Neste estudo, não se pode assumir a mesma argumentação desses autores ao atribuírem maior incidência de Aids entre homens de maior escolaridade, devido ao uso de drogas injetáveis ou à homossexualidade ser mais frequente entre eles.

A explicação mais plausível para a influência da escolaridade sobre informações que dependem de compreensão da doença parece ser o fato de que baixa escolaridade torna os indivíduos sem autonomia para buscar informações, para receber instruções mais complexas, produzir comunicações escritas de certa complexidade ou entender a mensagem que lhes é fornecida. São incapazes de aprender por conta própria, sem a tutela ou a mediação de professores ou instrutores, especialmente quando a informação requer algum grau de abstração ${ }^{28}$. Na medida em que as informações sobre Aids e HIV não perpassaram a escolarização dos idosos, assim como ainda hoje muitas vezes não são contempladas de forma científica na mídia e são transmitidas oralmente, eivadas de imprecisões, então se pode compreender o porquê desses resultados.

Para a melhoria da qualidade de vida da população, em especial dos idosos, faz-se necessá- 
rio promover campanhas e/ou programas de prevenção em relação às DST e ao HIV. A primeira iniciativa nacional nesta direção surgiu em 2008, quando a campanha do dia mundial de combate à Aids, promovida pelo Ministério da Saúde, teve como foco a prevenção da síndrome entre os idosos com o slogan "Sexo não tem idade, proteção também não”, porém muito ainda precisa ser feito, para que essa iniciativa possa ser continuada e consolidada.

Uma das limitações que se pode apontar neste estudo foi o uso de um critério único de satisfação com a vida, aplicado indistintamente para adultos jovens e idosos, deixando livre a cada sujeito o significado da expressão. Talvez fosse mais adequado o emprego de um questionário geral voltado para qualidade de vida, mas esse não foi o objetivo da pesquisa. Outra observação referese à diferença estatisticamente significante de algumas variáveis quando ajustadas por nível escolar, dado que o intervalo de confiança muito largo pode estar indicando uma inadequação da amostra para identificar essa influência por refletir possível baixo poder de aferição do teste.

Percebe-se, assim, a importância em reconhecer os valores e a cultura dos indivíduos envolvidos e, desse modo, promover campanhas com direcionamentos diferentes ao público jovem e aos idosos. Objetiva-se, com isso, atingir resultados mais efetivos em termos de prevenção, de uma atividade sexual segura, promovendo saúde à população de forma mais equânime, haja vista o contingente de idosos com esclarecimento sobre a doença, seu contágio e prevenção ainda estar muito aquém do encontrado entre os jovens.

\section{Colaboradores}

HMA Melo trabalhou na concepção do projeto, na análise dos dados e na redação do texto; MCC Leal aprovou a versão final, APO Marques auxiliou na construção do texto e na análise dos resultados e JG Marino revisão fez a crítica do texto sugerindo modificações. 


\section{Referências}

1. Fundação Instituto Brasileiro de Geografia e Estatística (IBGE). Projeção da População do Brasil por sexo e idade para o período de 1980-2050 - revisão 2008. Brasília: Fundação Instituto Brasileiro de Geografia e Estatística (IBGE), Departamento de população e indicadores sociais; 2008.

2. U.S. Census Bureau. Age data of the United States: special age groups. New York: US Census Bureau; 2008. [acessado 2009 jun 22]. Disponível em: http:/ /www.census.gov/population/www/socdemo/age/ general-age.html\#older

3. Lima-Costa M, Barreto S. Tipos de estudos epidemiológicos: conceitos básicos e aplicações na área do envelhecimento. Epidemiologia e Serviços de Saúde 2003; 12(4):189-201.

4. Barbosa A. Sexualidade. In: Saldanha A, Caldas C, organizadores. Saúde do idoso: a arte de cuidar. 2a ed. Rio de Janeiro: Interciência; 2004. p. 322-333.

5. Bassit A, Brandão D. Reflexões sobre a sexualidade feminina. In: Pacheco J, Py Ligia, Sá JL, organizadores. Tempo: rio que arrebata. São Paulo. 2005. p. 165-192.

6. Lindau ST, Schumm LP, Laumann EO, Levinson W, O'Muircheartaigh CA, Waite LJ. A study of sexuality and health among older adults in the United States. N Engl J Med 2007; 357(8):762-774.

7. Inelmen EM, Gasparini G, Enzi G. HIV/AIDS in older adults. A case report and literature review. Geriatrics 2005; 60(9):26-30.

8. Brasil. Ministério da Saúde (MS). Boletim Epidemiológico AIDS e DST 2008 V(1), 27-52 semanas epidemiológicas, julho a dezembro de 2007, 01-26 semanas epidemiológicas, janeiro a junho de 2008.

9. Stall R, Catania J. AIDS risk behaviors among late middle aged and elderly Americans. Arch Intern Med 1994; 154(1):57-63.

10. Alves FAP. AIDS e envelhecimento: características dos casos com idade igual ou maior a 50 anos em Pernambuco, 1990 a 2000 [tese]. Recife: Universidade Federal de Pernambuco; 2002.

11. Silva VTLB, Sá MLB. AIDS no panorama de um grupo de idosos. In: Anais do XIV Congresso Brasileiro de Geriatria e Gerontologia; 2004; Salvador.

12. Fonseca MG, Bastos FI, Derriço M, Andrade CLT, Travassos C, Szwarcwald CL. Aids and level of education in Brazil: temporal evolution from 1986 to 1996. Cad Saude Publica 2000; 16(Supl. 1):77-87.

13. Brasil. Lei no 8.842 de 4 de janeiro de 1994. Dispõe sobre a política nacional do idoso, cria o Conselho Nacional do Idoso e dá outras providências. Diário Oficial da União 1994; 5 jan.

14. Vieira EM, Villela WV, Réa MF, Fernandes MEL, Franco E, Ribeiro G. Alguns aspectos do comportamento sexual e prática de sexo seguro em homens do município de São Paulo. Cad Saude Publica 2000; 16(4):977-1009.

15. Batista AFO. AIDS: conhecimento sobre a doença entre idosos participantes da universidade aberta à terceira idade (UnATI)/Universidade Federal de Pernambuco (UFPE) [tese]. Recife: Universidade Federal de Pernambuco (UFPE); 2007.
16. Henderson S, Bemstein LB, George DM, Doyle JP, Paranjane AS, Corbie-Smith G. Older women and HIV: how much do they know and where are they getting their information? J Am Geriatrics Soc 2004; 52(9):1549-1553

17. Zablotsky D, Kennedy M. Risk factors and HIV transmission and older women: knowledge, options and the initiation of safer sexual practices. $J$ Acq Immun Defic Syndr 2003; 33:122-130.

18. Knodel J, Zimmer Z. Older persons Aids knowledge and willingness to provide care in an impoverished nation: evidence from Cambodia. Population Studies Center. Inst Soc Res. Michigan: University of Michigan; 2006.

19. Zornitta M. Os novos idosos com Aids e desigualdade à luz da bioética [dissertação]. Rio de Janeiro: Fundação Oswaldo Cruz (Fiocruz), Escola Nacional de Saúde Pública Sergio Arouca (ENSP); 2008

20. Figueiredo MAC, Provinciali RM. HIV/Aids em pessoas idosas. Vulnerabilidade, convívio e enfretamento. In: Anais do VII Congresso Virtual HIV/ AIDS. p. 8 [acessado 2007 jun 2]. Disponível em: http://www.Aidscongress.net/pdf/280.pdf

21. Gebo KA, Justice A. HIV infection in the elderly. Curr Infect Dis Report 2009; 11(3):246-254.

22. Lisboa MES. A invisibilidade da população acima de 50 anos no contexto da epidemia HIV/Aids. In: Anais do VII Congresso Virtual HIV/AIDS. [acessado 2007 jun 2]. Disponível em: http://www. Aidscongress.net/pdf/281.pdf

23. Zawacki T, Norris J, Hessler DM, Morrison DM, Stoner SA, George WH, Davis KC, Abdallah DA. Effects of relationship motivation, partner familiarity, and alcohol on women's risky sexual decision making. Pers Soc Psychol Bull 2009; 35(6):723-736.

24. Silva LS, Paiva MS, Carneiro AJS. Aids e masculinidade entre idosos. In: Anais do VII Seminário Fazendo Gênero; 2006; Florianópolis.

25. Geluda K, Bosi MLM, Cunha AJLA, Trajman A. "It takes two to tango" a study on inconsistent use of male condoms by adolescents in Rio de Janeiro, Brazil. Cad Saude Publica 2006; 22(8):1671-1680.

26. Guerriero I, Ayres JRCM, Hearst N. Masculinidade e vulnerabilidade ao HIV de homens heterossexuais, São Paulo, SP. Rev Saude Publica 2002; 36 (Supl. 4):50-60.

27. Berquó E, Barbosa RM, Lima LP. Trends in condom use: Brazil 1998 and 2005. Rev Saude Publica 2008; 42(Supl. 1):34-44.

28. Tafner P, editor. Brasil: o estado de uma nação 2006 - mercado de trabalho, emprego e informalidade. Rio de Janeiro: Instituto de Pesquisa Econômica Aplicada (IPEA); 2006.

Apresentado em 25/10/2009

Aprovado em 21/02/2010

Versão final apresentada em 15/03/2010 